\title{
Survey of Certain Properties of Repetitive Gain Scheduling Control Systems Using the Method of the Algebraic Derivative
}

\author{
E. Nikolov, N. G. Nikolova, B. Grasiani
}

Key Words: Repetitive gain scheduling control systems; methods for synthesis and analysis of performance; method of the algebraic derivative.

\begin{abstract}
The present study systematizes certain methods for analysis at priori un-certainty and proposes new methods for analy-sis of the filtration features of the repetitive gain scheduling control sys-tems under the conditions of periodic harmonic interference. For analysis of the fil-tering features of the repetitive gain scheduling control systems, the following methods have been proposed and applied to a particular example: temporal analy-sis with "test" periodic im-pacts in nomi-nal parametric mode and frequency analysis, using the method of the alge-braic derivative.
\end{abstract}

\section{Introduction}

There are control systems with parametric compensation (GSC Systems - Gain Scheduling Control Systems). The idea for this class of systems, realizing member of GSC-control strategy is proposed by J. Shamma, M. Athans $[1,2]$. It consists of introducing the additive compensation variables affecting basic management to compensation and parametric stabilization of transert gain of control systems. The analytical synthesis of these variables is based on previously known functional relationships of reparameterization (restructuring) in the model of control plant of measurable external disturbance signal. Based on a systematic study of these relationships with control valve $[3,4,5]$ strategy for gain scheduling control and its industrial applications $G S C$-systems developed in $[6,7,8,9]$. Repetitive control is an effective strategy for control and/or stabilization of industrial plant. The foundations of the idea and the principles of repetitive control placed by Normey-Rico Julio Elias [10], Eduardo F. Camacho [11], Ramon Costa-Castelly $[12,13]$, Liuping Wang [14,15]. Strategy repetitive control is designed to ensure high performance and maximum efficiency of the control systems, characterized in operating conditions with the presence of permanent periodic external signal interference; the parameters (time, frequency) are fixed and known in advance by value at the design stage of the system. The realization of repetitive control is performed using memory $\mathcal{M L}$-filters included series in the basic structure of the control algorithm. Principles of synthesis of repetitive $\mathcal{M L}$-control systems and their industrial applications are addressed systematically $[16,17,18]$.

\section{Objectiv and Tasks of Development}

The objective of this paper is to explore the possibilities of structural configuration and synthesis of a new class of control systems - systems with repetitive parametric compensation ( $\mathcal{M L} \circ G S C$-systems) and to analyze their performance and filtering properties being offered effective methods for this analysis. Implementation tasks to: analytic design of $G S C$-systems and $\mathcal{M L} \circ G S C$-control systems of a specific plant, a comparative performance analysis of the nominal parameter mode and robust performence frequency analysis of the synthesized systems.

\section{Formulation and Solution of the Problem of Structural Configuration}

Consider the structure (figure 1) of the traditional $[6 \div 9] G S C$-system with a fixed control algorithm $R_{G S C}$ for generalized plant $G$ in industrial operating conditions, where: the controller $R^{*} \underset{\{\sigma=c \text { const }\}}{\Leftrightarrow} G^{*}$ is optimally set in the context of the criterion $\sigma$ of nominal model $G^{*}$ of the object $G=G_{1} G_{2}$; embarrassed at the upper limit of the plant model $G \quad ; y$ - process control variable; $y^{0}$ - setpoint processing; $\varepsilon$ - system eroor; $\mu$ - control output processing of $G_{l}$; $q$ - volumetric flow rate for $G_{2} ; v$ - disturbance loading; $s$ - hydrodynamic loads to $G_{i} ; \xi$ - parametric disturbance in the process $G_{2} ; f$-perturbation to measurement; $\nabla \ell, \nabla a$ - compensating variables of GSC (1), where $\kappa_{\ell i}$ (2) control system transfer gain; $-s$ normalized hydraulic loading factor; $P^{0}$ - pressure supply. The analytical synthesis $[6 \div 9]$ of GSC -algorithmic (1) is independent of $G^{*}$ and $G^{\boldsymbol{m}}$, and is a function of (1) only by the type of control valve - linear ${ }^{(\text {lin })}$ or logarithmic ${ }^{(\log )}[3,4,5]$ after fixing the setting of $R^{*}(p) \underset{\{\sigma=\text { const }\}}{\Leftrightarrow} G^{*}(p)$. The designe method of $G S C$-algorithm (1) is ${ }^{\circ}$ compensatory parametric equation of balance $^{\circ}(3)$ at optimality criterion ${ }^{\circ}$ a constant value of the transfert gain of control system ${ }^{\circ} k_{\ell i, t}=$ const (3).

In this paper it is proposed structural configuration of a new class $\mathcal{M L} \circ G S C$-systems (figure 2), which unlike to 
GSC (figure 1) have the structure of the control algorithm $R_{M L \circ G S C}$ and $\mathcal{M L}$ (Memory Loop) memory [16 $\left.\div 18\right]$. By their nature, the latter is a bandpass filter, synthesized so that discarded (filtered cut maximum reduced damping) the impact of constant going external harmonic disturbance on the $d_{p}=\sin \left(\omega_{p} t\right)$ process control variable $y$. It is assumed that the frequency $\omega_{p}$ respectively the period $T_{p}$, are known in advance. Analytical synthesis $\mathcal{M L}$-filter is implemented in a consistent method for frequency correction ${ }^{\circ}$ rejecting module bandpass filter criterion ${ }^{\circ}$ (4) under criterion ${ }^{\circ}$ in horizontal profile module sensitivity-community ${ }^{\circ}$ of repetitive system $(5)[16 \div 18]$. The synthesis of the $\mathcal{M L}$-filter is independent of $G^{*}, R^{*}, G^{\boldsymbol{\square}}$ and $G S C$ is a function exclusively of the frequency $\omega_{p}$ of the disturbance $d_{p}$.

\section{Methods for Solution \\ of the Problem of Synthesis of $M \mathcal{L} \circ G S C$-Control Systems}

The stated in the previous section is the reason why this paper proposes a method for the analytical synthesis of $\mathcal{M L} \circ G S C$ control systems ${ }^{\circ}$ compensatory equation parametric balance and rejecting module bandpass filter ${ }^{\circ}$, summarizing dependencies (3) and (4) in integral optimality criterion ${ }^{\circ}$ constant value of the transfer gain and the horizontal profile of the module sensitivity of the control system ${ }^{\circ}$, summarizing (3) and (5). The work is available analytically designing a control algorithm $\mathrm{R}_{M \mathcal{M} \text { ○ GSC }}$ (figure 2) in three stages. The first task of deciding the optimal choice and adjustment of the basic regulator in the system $R^{*} \underset{\{\sigma=\text { const }\}}{\Leftrightarrow} G^{*}$. The second stage takes into account the type of control valve used in the system and analytical configured (1) both compensating variables $\nabla \ell, \nabla a$. The third stage, on the basis of (4), (5) and (6), consists in the synthesis of the $\mathcal{M L}$-filter and is connected with the choice of the structure of the filter and the number of units memory $e^{-p T_{p}}$ in her.

\section{Analytical Synthesis of $M \mathcal{L} \circ G S C$ - Control Systems. Numerical Example}

In this work was considered a numerical example in which it is necessary to synthesize the $G S C$-system (figure 1) and $\mathcal{M L} \circ G S C$-control system (figure 2) for the plant $G$ (figure 3 ) with the set $(7) \div(10)$ at a local optimality criterion ${ }^{\circ}$ critical aperiodic process ${ }^{\circ}(\sigma=$ const $)$ and integral criterion ${ }^{\circ}$ constant value of the transfer gain and the horizontal profile of the module sensitivity control system $^{\circ}(3),(5)$ in the presence of a permanently functioning periodic external signal interference $d$ with frequency $\omega_{p}=0,0346 \mathrm{rad} / \mathrm{s}(11)$.

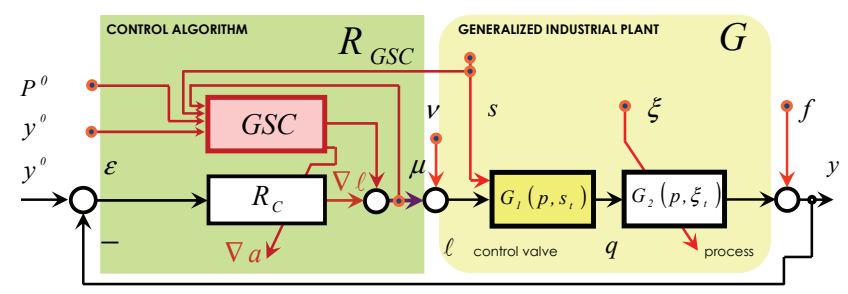

Figure 1

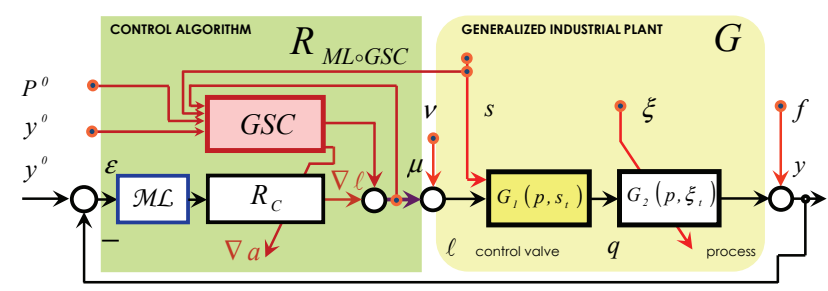

Figure 2

(1)

$$
\left\{\begin{array}{c}
G_{G S C}=\left[\begin{array}{c}
\nabla l \\
\nabla a
\end{array}\right]=\left[\begin{array}{ll}
c^{*} & C^{*}
\end{array}\right]\left[\begin{array}{c}
\nabla s \\
\nabla y
\end{array}\right], \\
c^{*}=-\frac{\kappa_{s_{0}^{*}}}{\kappa_{l_{0}^{*}}}\left(c_{l o g}^{*}=\frac{0.5\left(e^{2 n(1-l)}-1\right)}{n s e^{2 n(l-l)}} ; c_{l i n}^{*}=\frac{0,5\left(l^{-2}-1\right) l^{3}}{s}\right) \\
C^{*}=C_{l}^{*} ; \nabla y=\left(\frac{d y}{d l}\right)^{-1} \approx\left(\frac{\Delta y}{\Delta l}\right)^{-1}
\end{array}\right\}
$$

(2)

$$
\begin{gathered}
\kappa_{\ell i}=\frac{\partial q}{\partial l} \Delta l+\frac{\partial q}{\partial s} \Delta s ; \quad k_{i}=k_{I D}\left(\frac{\partial y}{\partial l} \Delta l+\frac{\partial y}{\partial s} \Delta s\right) ;\left(s=\left(1+\left(P^{0}-P_{1}\right)\left(P_{1}-P_{2}\right)^{-1}\right)^{-1}, \quad \nabla s=s_{i-1}-s_{i}\right) \\
k_{\ell i, t}=\text { const } \Leftrightarrow\left(R^{*}(\sigma)\right) ;\left(\frac{k_{l_{0}^{*}}\left(\Delta l+\nabla \ell_{t}\right)+k_{s_{o}^{*}}\left(\Delta s+\nabla s_{t}\right)}{d l}=\frac{k_{l_{0}^{*}} \Delta l+k_{s_{o}^{*}} \Delta s_{t}}{d l}\right)
\end{gathered}
$$




$$
\begin{array}{cc}
d\left|\mathcal{M}_{c, i}(j \omega)\right| / d \omega=0 & , \forall \omega \in \Delta \omega_{i} ; \\
\left|\mathcal{M}_{c, i}(j \omega)\right|=\text { const }<<1 & , \forall \omega \in \Delta \omega_{i} ; \\
\left|\mathcal{M}_{c, i}(j \omega)\right| \equiv 1 & , \forall \omega \in\left[0, \omega_{b, i}\right], \forall \omega \in\left[\omega_{h, i}, \infty\right) ; \\
\Delta \omega_{i} \in\left[\omega_{b, i}, \omega_{h, i}\right] & ,\left(\omega_{b, i}<\omega_{p}<\omega_{h, i}\right)
\end{array}
$$

(5) $\left|\mathcal{M}_{c, i}(j \omega)\right| \equiv\left\{\begin{array}{cc}0, & \forall \omega \in \Delta \omega_{i},\left(\omega_{b, i}<\omega_{p}<\omega_{h, i}\right) \\ 1, & \forall \omega \in\left[0, \omega_{b, i}\right], \forall \omega \in\left[\omega_{h, i}, \infty\right),\left(\omega_{b, i}<\omega_{p}<\omega_{h, i}\right)\end{array}\right.$

(6) $\mathcal{M}_{c, 1}(p)=\left(2-\prod_{q=2}^{n}\left(e^{-p T_{p}}\right)_{q}\right)^{-1}=\left(2-e^{-p q T_{p}}\right)^{-1}$

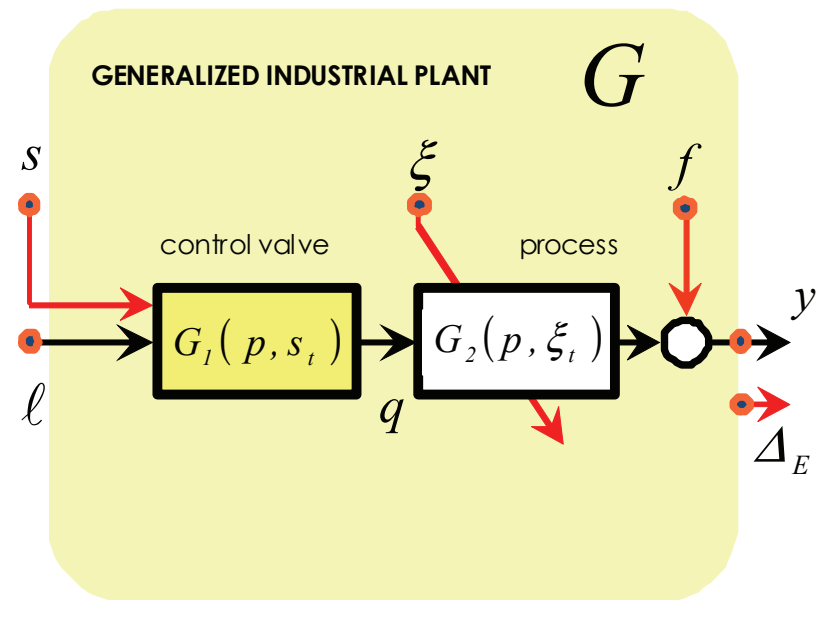

Figure 3a

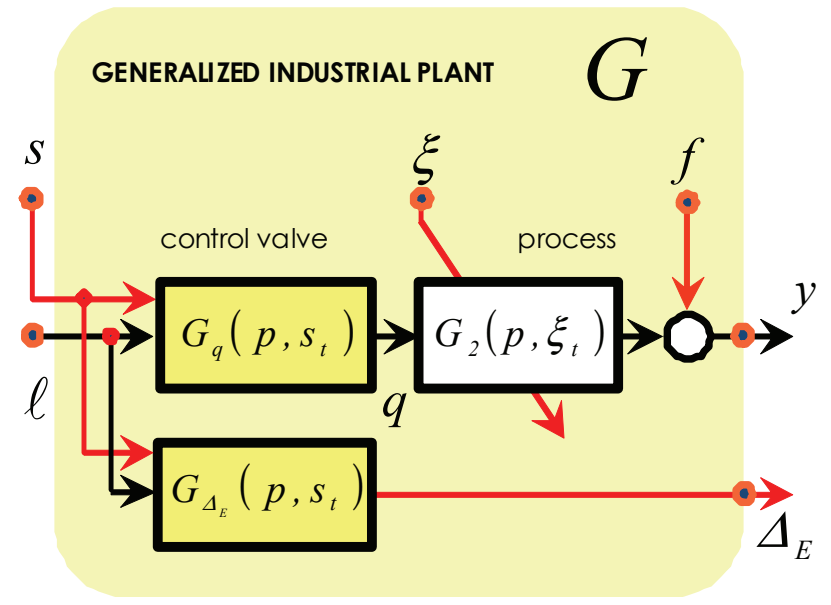

Figure 3b

(7)

$$
\begin{aligned}
& G^{*}(p, s)=G_{I}^{*}(p, s) G_{2}^{*}(p, \xi)=G_{q}^{*}(p, s) G_{2}^{*}(p, \xi)=\frac{y(p, s)}{\ell(p)} \\
& G^{*}(p, s)=\hat{G}^{*} \cdot e^{-\tau^{*} p}=\frac{y(p, s)}{\ell(p)} \\
& \hat{G}^{*}=\frac{(p+1)}{(1,6 p+1)} \cdot \frac{\left(1-s\left(1-e^{-8}\right)\right)^{-0,5}}{(0,04 p+1)}\left(\frac{15}{(4 p+1)\left(9 p^{2}+3 p+1\right)}\right) \\
& \left(s=0,00315, \xi=\text { const }, e^{-\tau p} \hat{=}(1+(p \tau / 3))^{-3} \hat{=}(1+p)^{-3},\left(\tau=\tau^{*}=3, s\right)\right)
\end{aligned}
$$

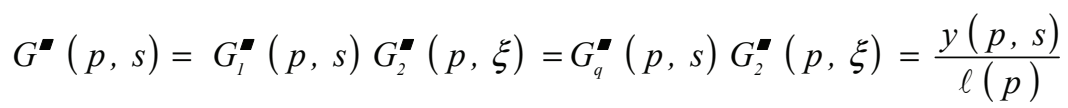

$$
\begin{aligned}
& G^{\bullet}(p, s)=\hat{G}^{\boldsymbol{\bullet}} \cdot e^{-\tau^{*} p}=\frac{y(p, s)}{\ell(p)} \\
& G^{\mathbf{\Delta}}(p)=\frac{(p+1)}{(1,6 p+1)} \cdot \frac{\left(1-s\left(1-e^{-8}\right)\right)^{-0,5}}{(0,04 p+1)}\left(\frac{15}{(4 p+1)\left(9 p^{2}+3 p+1\right)}\right) \cdot(1+1,66 p)^{-3} \\
& (s=0,815, \xi=\text { const })
\end{aligned}
$$

(8)

(9)

$$
G_{q}(p, s)=\frac{q(p)}{\ell(p)}=\frac{\left(a\left(w_{0}\right) p+1\right)}{\left(b\left(w_{0}(t)\right) p+1\right)} \cdot \frac{\left(1-s\left(1-\sigma^{-2}(\ell)\right)\right)^{-0,5}}{(T(s) p+1)}
$$




$$
G_{\Delta_{E}}(p)=\frac{\Delta_{E}(p)}{\ell(p)}=c \cdot s \cdot \frac{\left(a\left(w_{0}\right) p+1\right)}{\left(b\left(w_{0}(t)\right) p+1\right)} \cdot \frac{\left(1-s\left(1-\sigma^{-2}(\ell)\right)\right)^{-0,5}}{(T(s) p+1)}
$$

$$
d=\sin \left(\omega_{p} t\right)=\sin (0,0346 t)=\mathrm{const},\left(\omega_{p}=\omega_{p f}=0,0346 \mathrm{rad} / \mathrm{s} ; T_{p f}=181,595 \mathrm{~s}\right)
$$$$
R *(p)=\frac{2(2 p+1)}{2 p} \frac{(p+1)}{(0.2 p+1)}, \quad\left(R^{*} \underset{\{\sigma=\text { const }\}}{\Leftrightarrow} G^{*}\right)
$$

$$
\nabla \ell_{t}=-\frac{0,5\left(l^{-2}-1\right) l^{3}}{s} \nabla s_{t} ; \nabla a_{t}=c_{0}^{*}\left(\frac{d y_{t}}{d \ell_{t}}\right)^{-1}
$$

$$
\mathcal{M}_{L}(p)=\left(2-\sum_{k=1}^{m} W_{k}(p) e^{-p k T_{p}}\right)^{-1} \hat{\equiv}\left(2-e^{-p T_{p}}\right)^{-1},\left(k=1 ; T_{p} \equiv T_{p f}=2 \pi\left(\omega_{p f}\right)^{-1}=2 \pi(0,0346)^{-1}\right)
$$

$$
\alpha_{i, G S C}^{\left(\omega_{i}\right)}(\omega) \hat{\equiv} \frac{d e_{G S C}(\omega)}{d \omega}=\frac{d\left(\left(1+R_{G S C}(j \omega) G^{*}(j \omega)\right)^{-1}\right)}{d \omega} \equiv \frac{d\left(\Phi_{y^{\circ}}^{G S C}(\omega)\right)}{d \omega}
$$

$$
\left.\alpha_{i, M L \circ G S C}^{\left(\omega_{i}\right)}(\omega)\right|_{\omega=\omega_{p}}<<\left.\alpha_{i, G S C}^{\left(\omega_{i}\right)}(\omega)\right|_{\omega=\omega_{p}}
$$

$$
\left.\frac{d e_{M L \circ G S C}(\omega)}{d \omega}\right|_{\omega=\omega_{p}}<\left.\frac{d e_{G S C}(\omega)}{d \omega}\right|_{\omega=\omega_{p}}
$$

In the analytical description of the plant: $\Delta_{E}$ are hydrodynamical energy losses; $w_{o}$ - initial velocity of fluid throttle; $\sigma^{-2}(\ell)$ - some theoretical transmission characteristic control valve; $a, b, T-$ known design constants control valve.

The characteristics of the given numerical example in plant are shown in figure 4. Based on the systematized analytical methods for synthesis and set optimality criteria, solving the problem of analytical synthesis of the $G S C$-system (figure 1) and $\mathcal{M L} \circ G S C$ - control system (figure 2) set with $(7) \div(10)$ plant (figure 3), defines the main algorithmic components of $R_{G S C}$ (figure 1) and $\mathrm{R}_{M \mathcal{M L G S C}}$ a (figure 2) as shown at (12) $\div$ (14). The characteristics of $R_{G S C}, \mathbf{R}_{\mathscr{M L} \circ G S C}$ and the mechanism of compensating variables $\nabla \ell, \nabla a$ are illustrated in figure 5 .

\section{Solution of the Problem for Analysis}

\subsection{Analysis of Performance in Nominal Parameter Mode}

Both systems (figure 1, figure 2) are modeled. The results of the simulation models - timing and frequency characteristics of the decision $(12) \div(14)$ for closed loop $\Phi_{i}$ and open loop $W_{i}$ parametric compensation system and repetitive parametric compensation $\mathcal{M L} \circ G S C$-control sys-tem specified by (7) $\div$ (10) plant (figure 3) are shown in figure 6, figure 7. The characteristics of the systems are displayed in nominal (7) parameter mode ( $G \hat{\equiv} G^{*}, \xi=$ const $, d_{i} \equiv 0, s=0,00315=$ const $)$. 
Used were the following: • indexes systems: parametric compensation $G S C$-system (figure 1), repetitive gain scheduluing control system $\mathcal{M L} \circ G S C$ (figure 2) • indications: $h_{\Phi_{i}}-$ transfer functions, $\Phi(j \omega), W(j \omega)$ - frequency characteristics of closed and open systems, $e(j \omega)$ - characteristics of the sensitivity of the closed systems.

Performance analysis in nominal (7) parameter mode
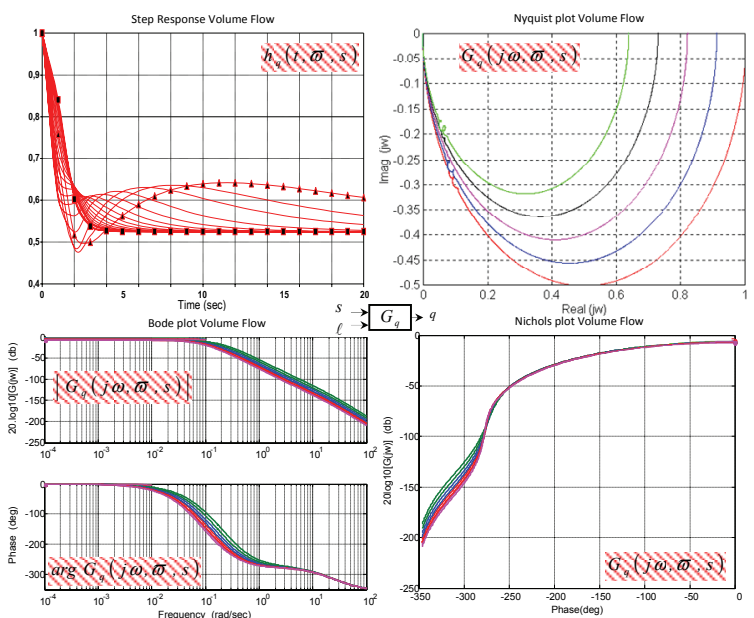

Figure 4a
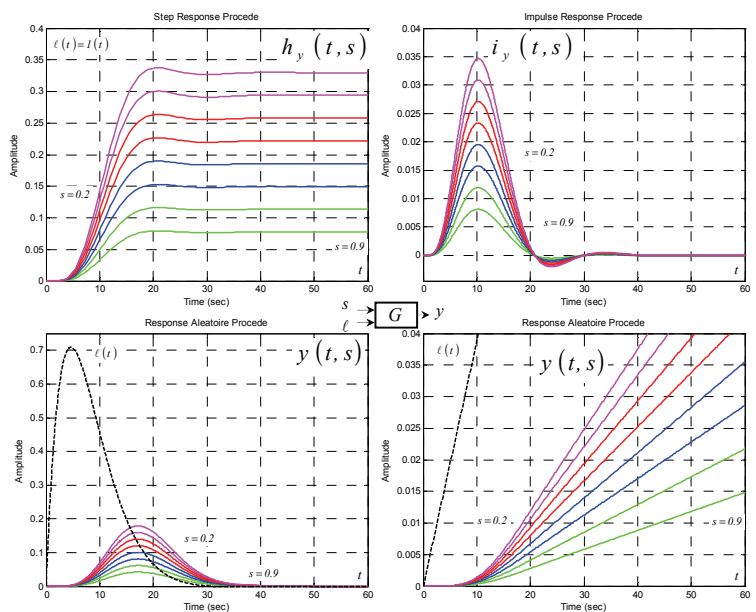

Figure 4c
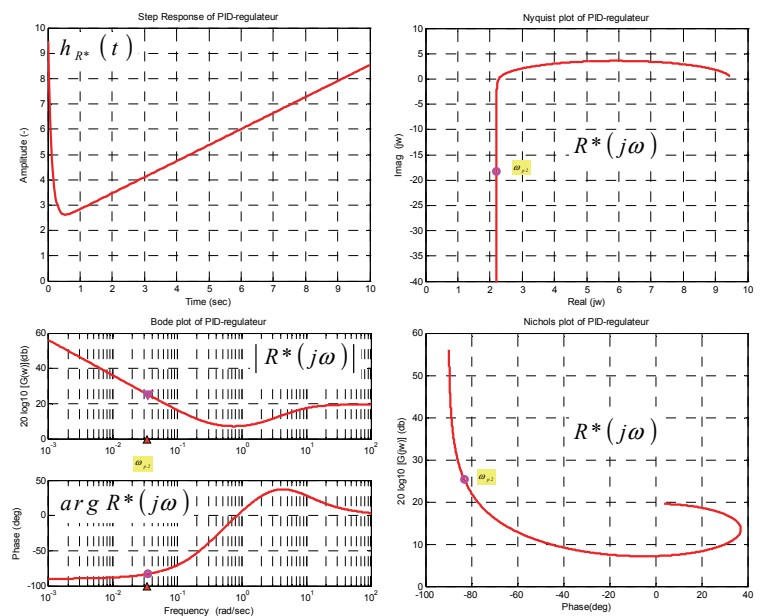

Figure 5a systems (figure 1, figure 2) confirms that both systems: • meet the criteria for ${ }^{\circ}$ critical aperiodic process $^{\circ}(\sigma)$ (figure 6 ) and is provided an opportunity to report graphically times regulation setting fast response; - stability (figure 7), it has provided an opportunity to report their gaine marge and phase marge of stability; $\bullet$ claimed satisfy performance requirements in the process of synthesis.
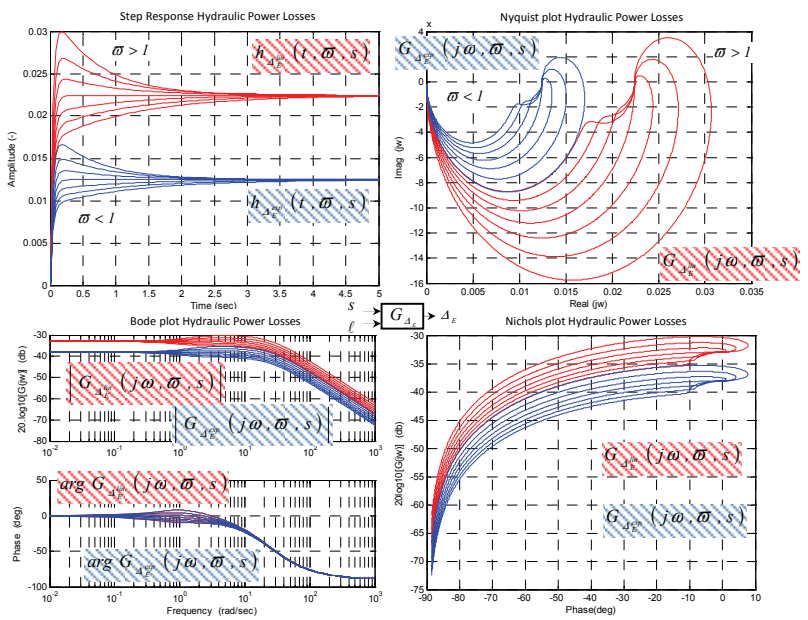

Figure 4b
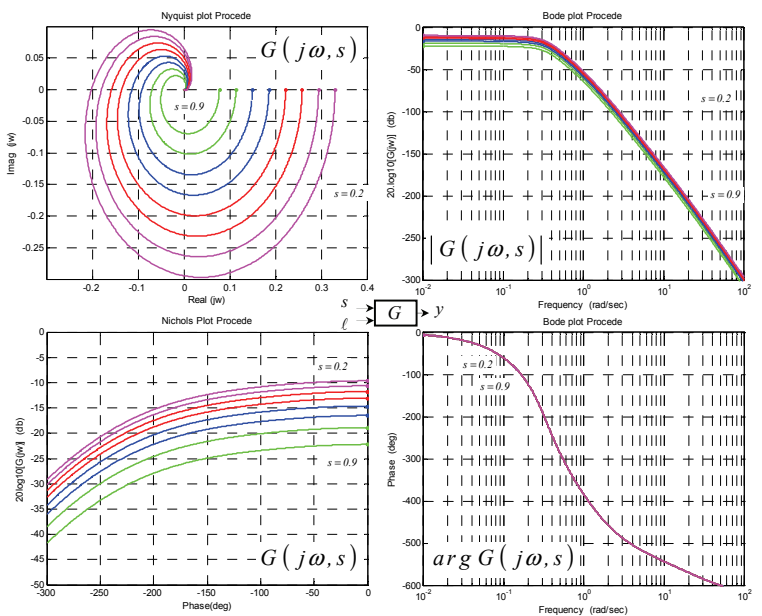

Figure 4d
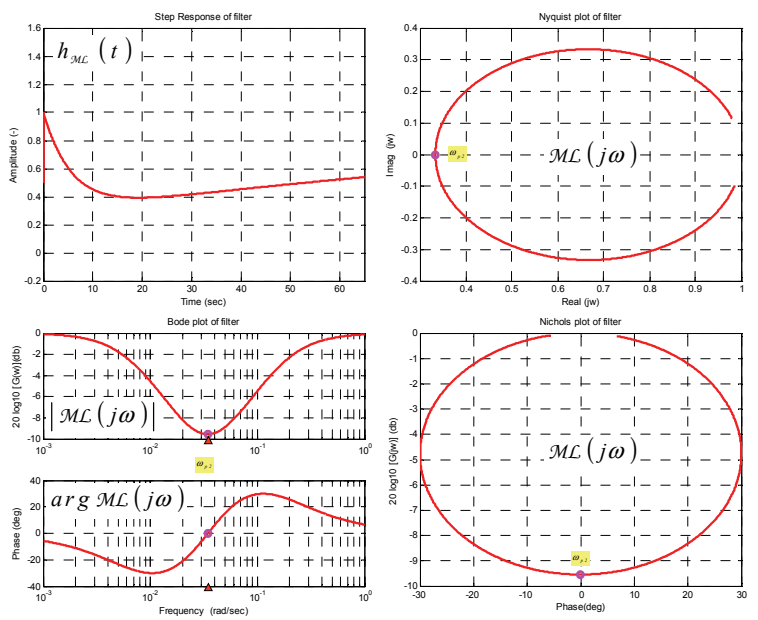

Figure 5b 

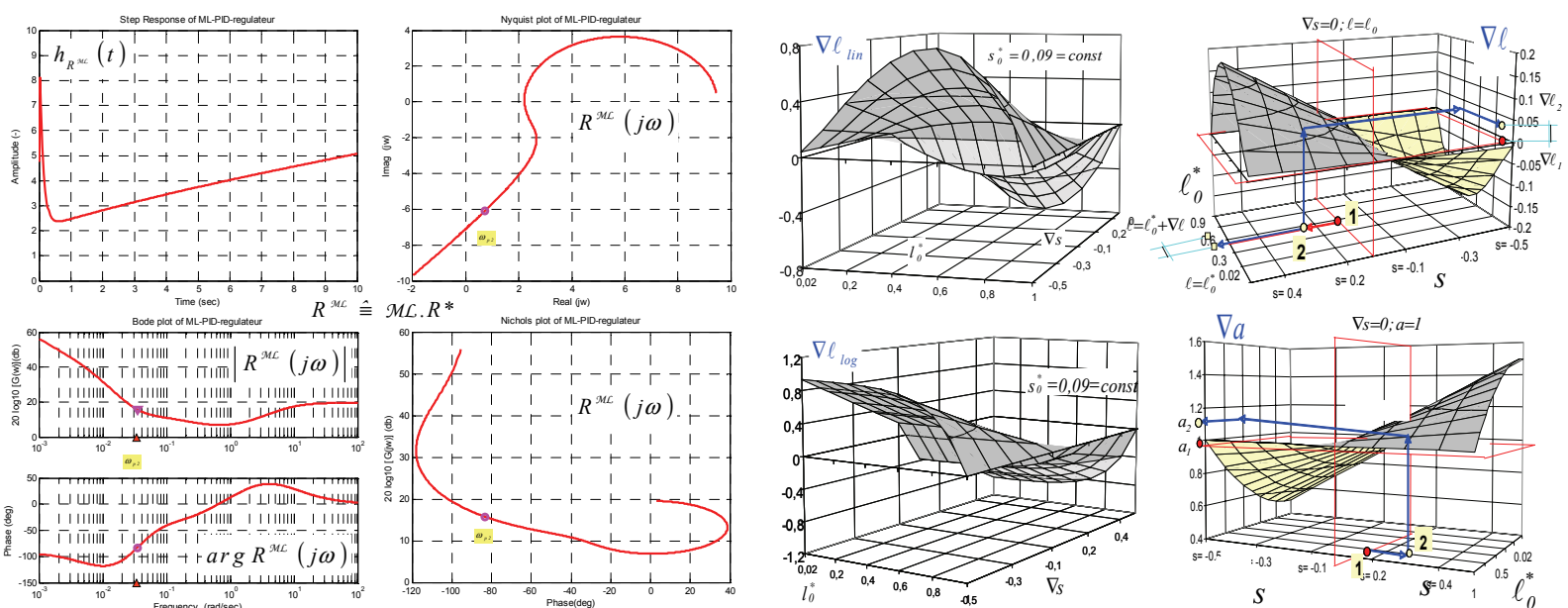

Figure 5c

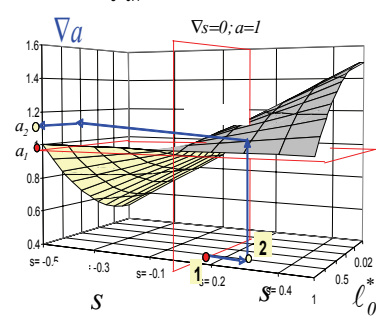

Figure 5d
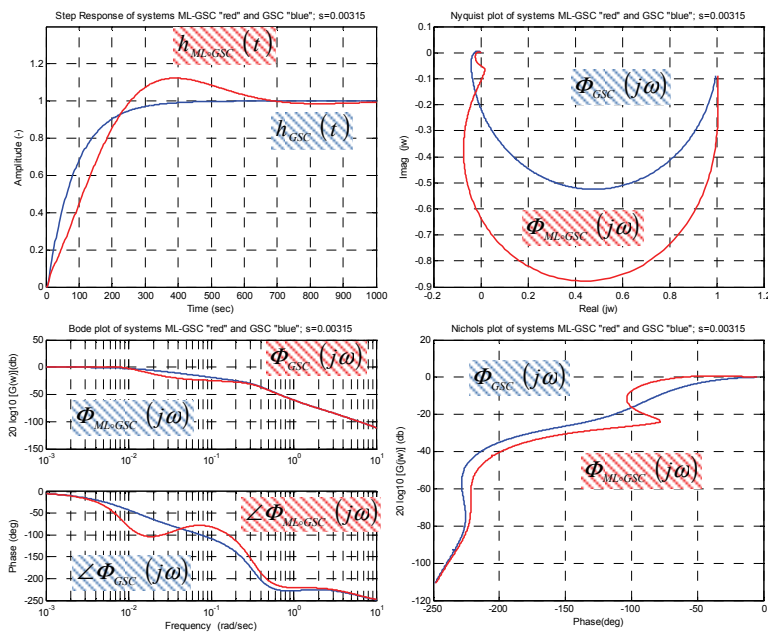

Figure 6
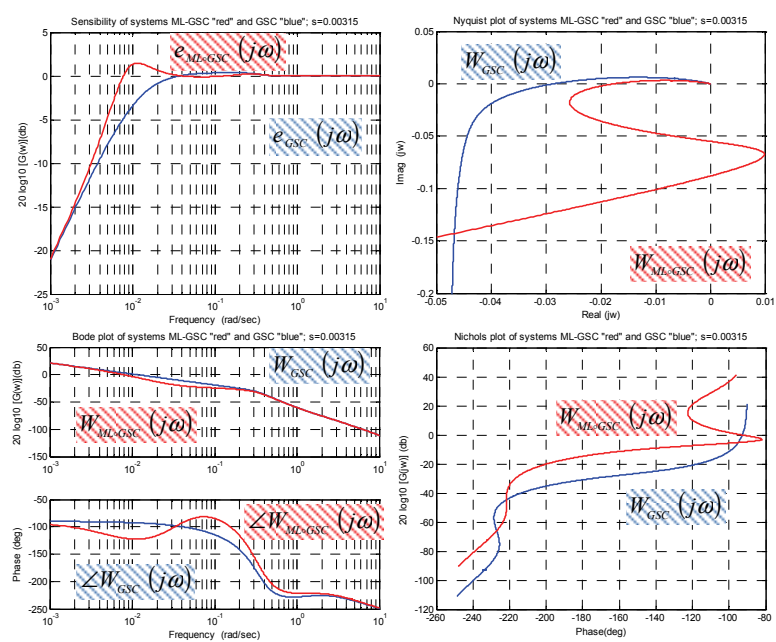

Figure 7 


\subsection{Temporal Analysis of Filtering Properties}

The analysis was conducted of the decision $(12) \div(14)$ at a constant acting periodic external signal disturbance $d$ with frequency $\omega_{p}(11)$. Disturbance is applied parallel to the synthesized models of both control systems (figure 1, figure 2) set with $(7) \div(10)$ plant $G$ (figure 3 ) in conditions of external perturbation is an analysis of the decision $(12) \div(14)$ at a constant acting periodic external signal per-turbation with frequency (11). The time analysis of the filtering properties, designed to explore combating repetitive systems of external impact, consider the parallel behavior of the two systems (figure 1, figure 2) impact of periodic $d$ (11) submitted to the canal systems: the assignment, parallel assignment and load measurement, parallel assignment and measurement parallel on the job and load and measurement.

Under these conditions, the time analysis of the filtering properties to "test" periodic effects systems (figure 1, figure 2) was conducted in nominal (7) parameter mode. The essence of the method of analysis time to "test" effects consists in modeling the system and evaluating the simulation results on time criteria. Both systems (figure 1, figure 2) are modeled on the transfer functions $h_{i}(t)$ of the recurrent impact $d(11)$ are simulated. Weather characteristics of systems (hereinafter indicated by $h_{G S C}$ and $\left.h_{M \mathcal{L} \circ G S C}\right)$ in parallel as results of the simulation are illustrated in figure $8 \div$ figure 10 as follows of:

- Figure 8 - Disturbance with $d$ (11) in $y^{0}$;

- Figure 9-Disturbance with $d$ (11) parallel in $y^{0}$ and $d$ (11) the channel load $v$;

- Figure 10 - Disturbance to $d$ (11) in $f$;

- Figure 11 - Disturbance to $d$ (11) parallel in setpoint processing $y^{0}$ and $d(11)$ in $f$;

- Figure 12 - Disturbance to $d$ (11) parallel in setpoint processing $y^{0}$, and $d(11)$ in the groove of the load $v$ and with $d(11)$ in the measurement channel $f$;

- Figure 8a, figure 9a, figure 10a, figure 11a, figure $12 a$ - at external interference (11) and hydrodynamic load in control valve $s=0,00315=$ const;

- Figure 8b, figure 9b, figure 10b, figure 11b, figure $12 b$ - at external interference $d(11)$ and hydrodynamic load control valve $s=0,005315=$ const;

- Figure 8c, figure 9c, figure 10c, figure 11c, figure $12 c$ - at external interference $d(11)$ and hydrodynamic load control valve $s=0,007315=$ const;

- Figure 8d, figure 9d, figure 10d, figure 11d, figure $12 d$ - at external interference $d(11)$ and hydrodynamic load control valve $s=0,01315=$ const.

The results of the study by the method of analysis of temporal filtering properties of systems with "test" effects the following conclusions (in terms of quantitative assessments of the performance of processes - overshoot, overshoot number, time adjustment):

- repetitive gain scheduling control system $\mathcal{M L} \circ G S C$ (figure 2) is invariant to the harmonious effect $d$ (11), for which it is designed, which is especially pronounced in cases where $d(11)$ is channel assignment, load parallel and task and a load;

- for all research cases discussed and illustrated in figure $8 \div$ figure 12 there is no reference value that repetitive gain scheduling control system $-\mathcal{M L} \circ G S C$ (figure 2) not superior parametric compensation system GSC (figure 1);

- in terms of quantitative assessments of performance to repetitive gain scheduling control system $-\mathcal{M L} \circ G S C$ (figure 2) outperforms GSC gain scheduling control system - (figure 1) efficiency to counteract the constant perturbation with frequency $\omega_{p}=0,0346 \mathrm{rad} / \mathrm{s}$ (11).

\subsection{Frequancy Analysis Filtering Properties with Algebraic Derivatives}

For analytical reflection, testing and comparative evaluation of the properties of the filter systems, the use of a method of frequency $\alpha_{i}^{(\omega)}$ analysis using the algebraic derivative [16] in the direction of sensitivity of the system (figure 1, figure 2) in the nominal (7) parameter mode. It is an analytical method and consists in the effective use of the algebraic derivative [16] in a direction $\alpha_{i}^{\left(\omega_{l}\right)}$ defined by (15), (16) for each of the analyzing system (figure 1, figure 2). In comparison in figure 13 and figure 14 are shown the characteristics of sensitivity and algebraic derivatives (15) (16) system (figure 1, figure 2).

The analysis of the algebraic values of the derivatives (figure 14) in the direction of the sensitivity of the compared systems unambiguously prove that they are in a ratio of (17) corresponding to the reflected (18). The analytical method of frequency analysis of the filtering properties of systems using algebraic derived from the direction of the sensitivity of the system is a tool to quantify the filtering properties of the repetitive systems control. With his help determine:

- meet the criteria for treatment with the presence of ${ }^{\circ}$ horizontal profile of the module sensitivity ${ }^{\circ}$ beforehand to set frequency value $\omega_{i}=\omega_{p}(11)$;

- analytical criterion (5) satisfaction of ensuring the implementation of the filtering requirements of periodic external effects $d$ with frequency $\omega_{i}=\omega_{p}(11)$ of repetitive system;

- criterion benchmarking of filtering properties of systems control by defining the value of the algebraic derivatives (15) (16), illustrated in figure 14;

- in comparative terms in their filtering properties repetitive gain scheduling control system $-\mathcal{M L} \circ G S C$ ) proved superior in the context of (5) gain scheduling control system - GSC (figure 1).

The analytical method of frequency analysis of the filtering properties of systems using algebraic derived from the direction of the sensitivity of the system is a tool to quantify the filtering properties of the repetitive systems control. With his help determine:

- meet the criteria for treatment with the presence of ${ }^{\circ}$ horizontal profile of the module sensitivity ${ }^{\circ}$ beforehand to set frequency value $\omega_{i}=\omega_{p}(11)$;

- analytical criterion (5) satisfaction of ensuring the 


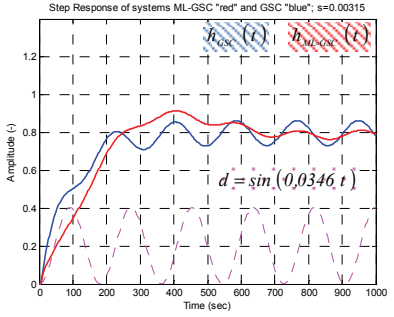

Figure 8a

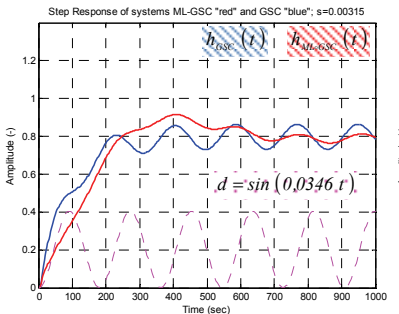

Figure 9a

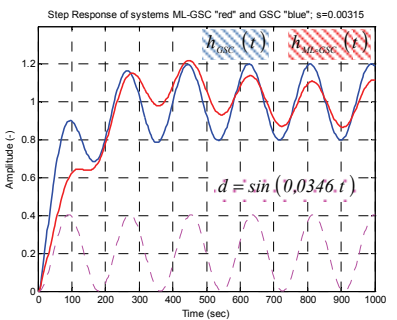

Figure 10a

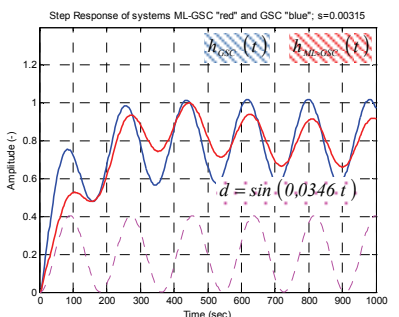

Figure 11a

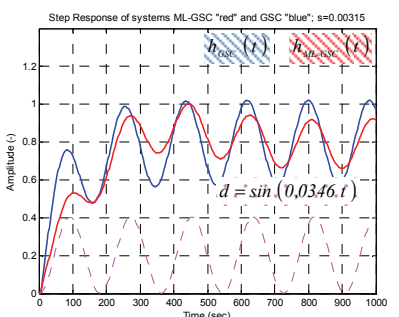

Figure 12a

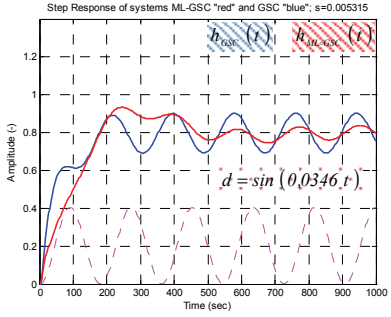

Figure 8b

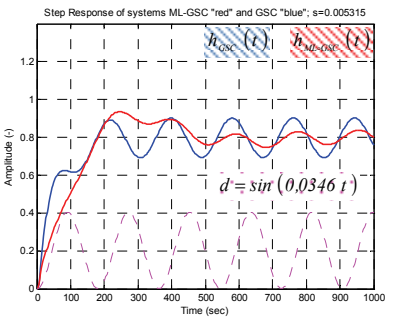

Figure 9b

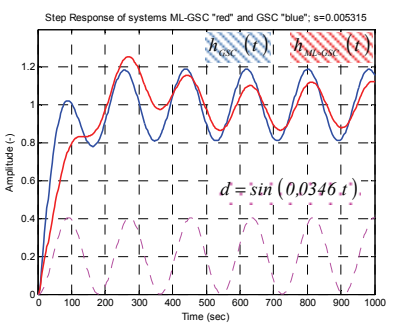

Figure 10b

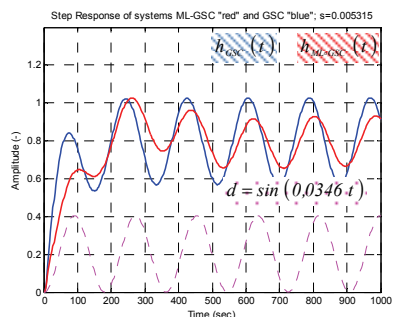

Figure 11b

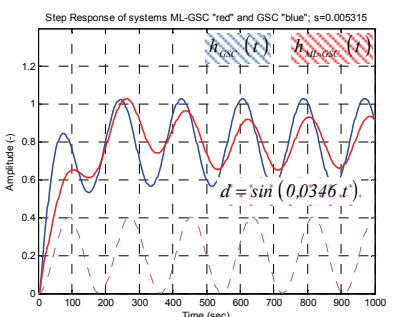

Figure 12b

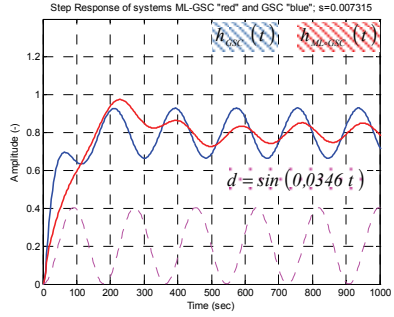

Figure 8c

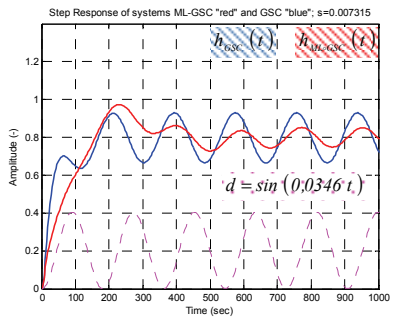

Figure 9c

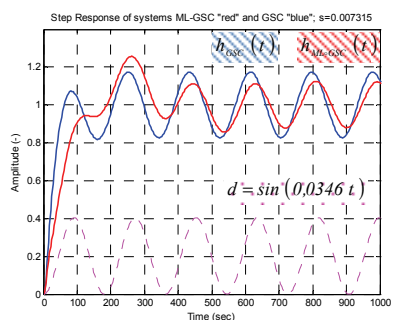

Figure 10c

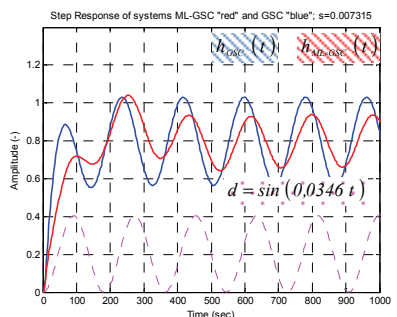

Figure 11c

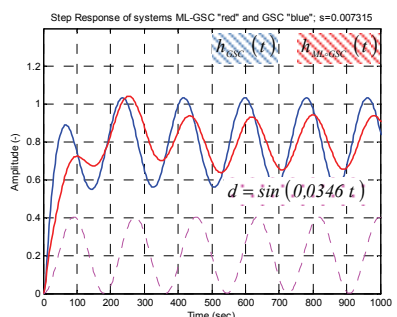

Figure 12c

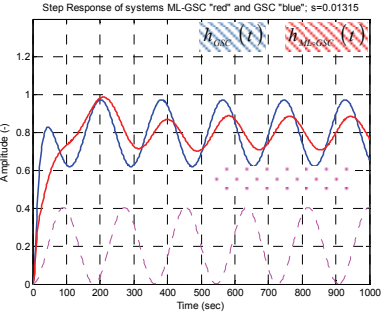

Figure 8d

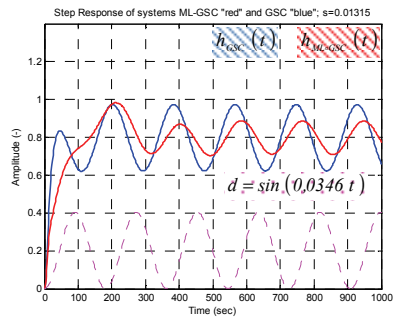

Figure 9d

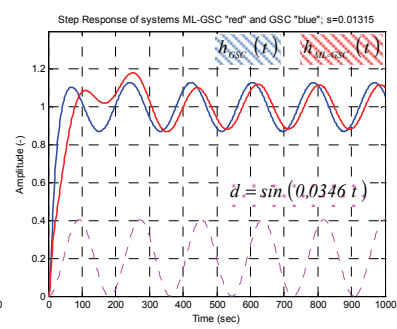

Figure 10d

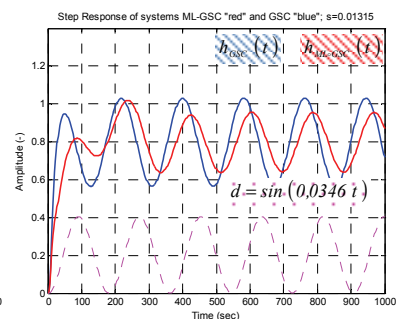

Figure 11d

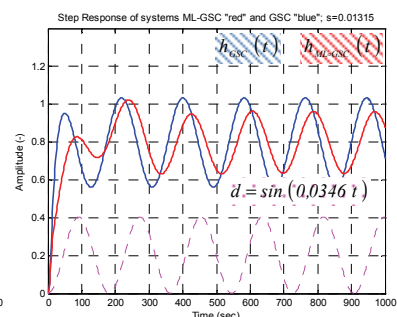

Figure 12d 

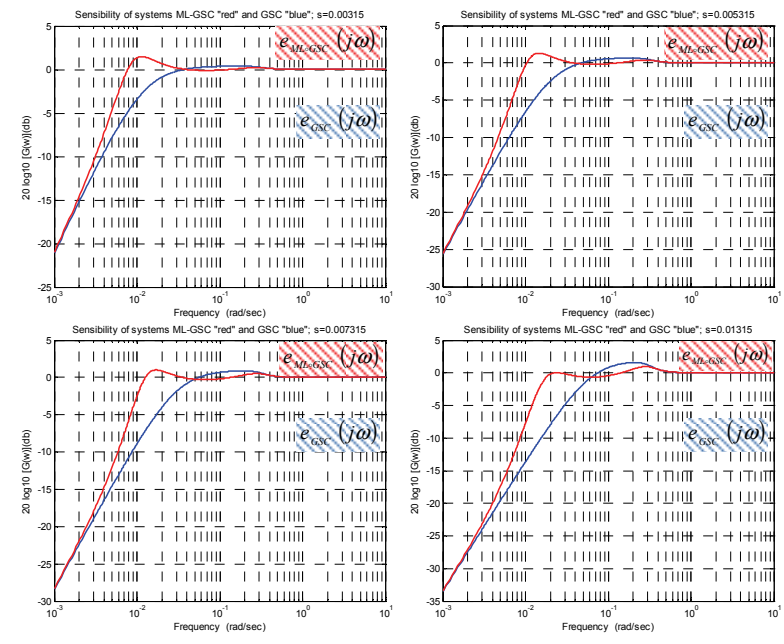

Figure 13

implementation of the filtering requirements of periodic external effects $d$ with frequency $\omega_{i}=\omega_{p}(11)$ of repetitive system;

- criterion benchmarking of filtering properties of systems control by defining the value of the algebraic derivatives (15) (16), illustrated in figure 14;

- in comparative terms in their filtering properties repetitive gain scheduling control system $-\mathcal{M L} \circ G S C$ ) proved superior in the context of (5) gain scheduling control system - GSC (figure 1).

\section{Analysis of the Decision}

The analysis of the survey results and the performence of the filtering properties of the synthesized control systems (figure 1, figure 2) of the plant $(7) \div$ (10) was held by three methods. Each of them proved that repetitive gain scheduling control system $-\mathcal{M L} \circ G S C$ satisfies the criteria brought in its synthesis by applying the method of:

- performance analysis in nominal (7) parameter mode systems (figure 1, figure 2) confirmed that both systems: the criteria for ${ }^{\circ}$ critical aperiodic process ${ }^{\circ}(\sigma)$, are stable, but the $\mathcal{M L} \circ G S C$ system (figure 2) at one the same other terms and superior GSC - system (figure 1) a fast response and the stability marge;

- time analysis of filtering properties of systems with "test" impacts and assessment of transitional functions (figure $8 \div$ figure 12 ) confirmed that the $\mathcal{M L} \circ G S C$ - system (figure 2) satisfies the criterion in the design of the filter for horizontal profile of the module sensitivity (4);

- frequency analysis of the filtering properties of systems using algebraic derivative in the direction of the sensitivity of the system (figure 14) in nominal (7) parameter mode was shown to $\mathcal{M L} \circ G S C$-system (figure 2) satisfies the criterion (4) filtering periodic external effects $d$ with frequency $\omega_{i}=\omega_{p}(11)$.

\section{Conclusion}

New and original in this paper are:

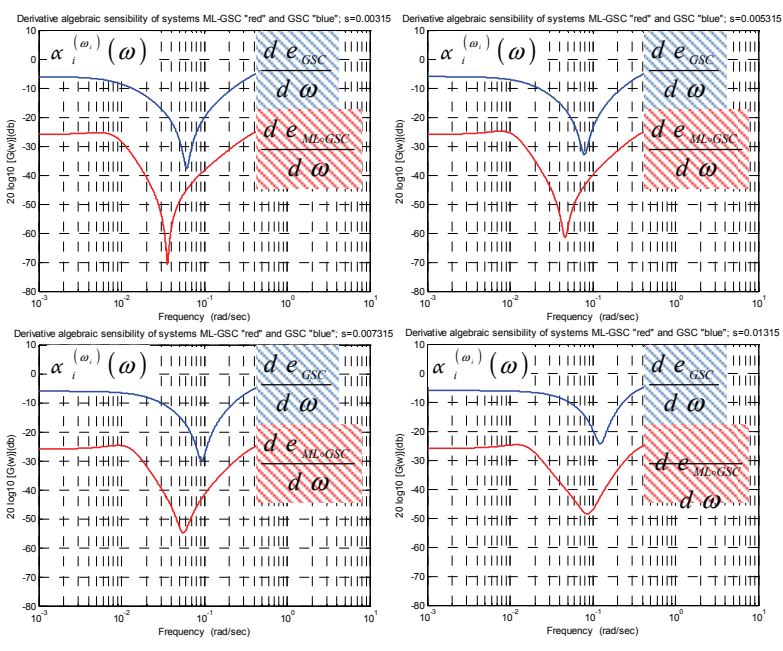

Figure 14

- configuring and the designe method of a new class of repetitive gain scheduling control systems;

- proposed methods for performence analysis and filtering properties of gain scheduling control system and repetitive gain scheduling control that are classified as methods:

- performence analysis in nominal parameter mode;

- temporal analysis of filtering properties to "test" periodic impacts in nominal parameter mode;

- frequency analysis of the filtration properties using alge-braic derivative in the direction of the sensitivity;

- results of the application of the proposed methods for performance assessment and filtering properties of control systems.

\section{References}

1. Shamma, J., M. Athans. Gain Scheduling: Potential Hazards and Possible Remedies. Proceed. American Contr. Conf., 1991, 516-521. 2. Shamma, J., M. Athans. Guaranteed Properties for Nonlinear Gain Scheduled Control Systems. Proceedings of the 27th Conference on Decision and Control, 1988, 2202-2208.

3. Nikolov, E. Control Instrumentations - part II (Control Algorithms, Intelligent Actuator, Noise Reduc-tion Control Valves). Ad. of Technical University of Sofia, Sofia, 2-nd Edd., ISBN 954438-336-6, 2003, 296.

4. Nikolov, E., N. G. Nikolova. Models and Analysis of the Smooth Flow Control in the Control Valves - Stationary Mode, Power Losses - Part I. - Journal Proceedings of the Technical University of Sofia, Publishing House of Technical University of Sofia, ISSN 0374-342X, ISSN 1311-0829, 63, 2013, 3, 53-62.

5. Nikolov, E., N. G. Nikolova. Models and Analysis of the Smooth Flow Control in the Control Valves - Non-Stationary Mode, Energy Losses - Part II. - Journal Proceedings of the Technical University of Sofia, Publishing House of Technical University of Sofia, ISSN 0374-342X, ISSN 1311-0829, 63, 2013, 4, 53-62.

6. Nikolov, E. Applied Methods for Process Control - part I (Frequency Methods and Systems with Robust Performances). Ad. of Technical University of Sofia, 2-nd Edd., ISBN 954-438-334-4, 2003, 358.

7. Nikolov, E., N. G. Nikolova. Application of Generalized Fractional Calculus in the Gain Scheduled Control Systems. - Journal Cybernetics and Information Technologies, 13, 2014, No. X, ISSN 
1311-9702, Bulgarian Academy of Sciences, 21-32.

8. Nikolova, N., E. Nikolov. Gain Scheduled Disturbance Absorbing Robust Control Systems with Internal Model. Proc. of International Conference Automatics and Informatics '2002, Bulgaria, 30-31 October 2003, Symposium "Control Power Plants and Systems", John Atanasoff Society of Automatics and Informatics, ISBN 954-9641-37-6, 3, 2003,15-18.

9. Nikolova, N., E. Nikolov. Gain Scheduled Robust Control Systems with Internal Model. Proc. of International Conference Automatics and Informatics'2002, Bulgaria, Session "Control Power Plants and Systems", 14-15 November 2002, John Atanasoff Society of Automatics and In-formatics, 3, 2002, 17-22, ISBN 954-9641-32-5.

10. Álvarez, J. D., J. L. Redondo, E. Camponogara, Normey-Rico Julio Elias, M. Berenguel, P. M. Ortigosa. Optimizing Building Comfort Temperature Regulation via Model Predictive Control. Energy and Buildings, Elsevier, 57, February 2013, 361-372.

11. Normey-Rico Julio Elias, Eduardo F. Camacho. Control of DeadTime Processes. Berlin, Spinger, 2007, 216.

12. Griñó Robert, Ramon Costa-Castelló. Digital Repetitive Plug-in Controller for Odd-harmonic Periodic References and Disturbances. - Automatica, 2007 Elsevier, 41, January 2005, Issue $1,153-157$.

\section{Manuscript received on 27.01.2016}

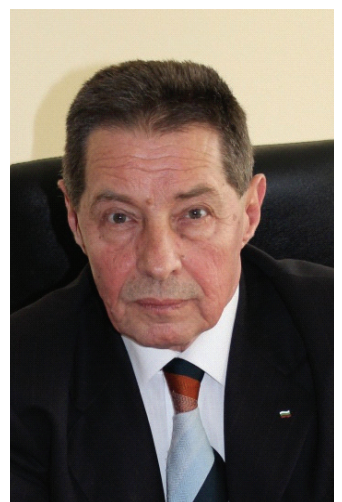

Prof. Emil Nikolov, D.Sc. Ph.D. (born 1950). Education: M.Sc. (1973) Technical University of Sofia. Scientific degrees: Ph.D. (1985) "Multivariable Control Systems - Analysis and Synthesis"; D.Sc. (1999) "Robust Control Systems". Employment: Assistant Prof. (1974-1978); Senior Assistant Prof. (1979-1987); Associated Prof. (19881999); Full Professor Technical University of Sofia, Faculty of Automation (2000 - present); Professeur des Universités I-ere classe de l'Université d'Artois - Faculté de Sciences Appliquées, France (2001 - present). Career related activities: Head of Department "Industrial Automation", Technical University of Sofia, Faculty of Automation (1995-2011); Head of Chaire UNESCO TUS (2004-2008); Dean of Faculty of Automation, Technical University of Sofia, Faculty of Automation (2011-2015). University teaching experience: (Technical University of Sofia - Faculty of Automation and Filière Francophone de Génie Electrique; l'Université d'Artois - Faculté de Sciences Appliquées, France); Scientific director of laboratory "Control Instrumentation and industrial automation" (1995-2015). Scientific interests: Fractional Control Algorithms and Systems; Robust- and Disturbances Absorbing Control and Industrial Applications; Control Instrumentation; Intelligent Control Instrumentation; Control Valves. Member of: John Atanasoff Society of Automatics and Informatics, IEEE, AFCEA, IFAC; Editorial Board of the International Journal of Automation and Control (IJAAC) Undersciences Publishers; Publications in international and Bulgarian journals - 450; books - 40 .

Contacts:

Technical University of Sofia Faculty of Automation

Department "Industrial Automation" e-mail: nicoloff@tu-sofia.bg
13. Gricy, R., R. Cardoner, Ramon Costa-Castelló, E. Fossas. Digital Repetitive Control of a Three-phase Four-wire Shunt Active Filter. - IEEE Transactions on Industrial Electronics, 54, 2007, 3 , 1495-1503.

14. Liuping Wang, Chris T. Freeman, Shan Chai, Eric Rogers. Predictive-repetitive Control with Constraints: from Design to Implementation. - Journal of Process Control, Elsevier, 23, 2013, 956-967.

15. Liuping Wang, S. Chai, Eric Rogers. Predictive Repetitive Control Based on Frequency Decomposition. American Control Conference, Baltimore, USA, 2010, 4277-4282.

16. Nikolova, N., E. Nikolov. Predictive Repetitive Control (Applied Methods for Process Control - Part III). Publishing House of Technical University of Sofia, ISBN: 978-619-167-136-6, 2014, 160.

17. Nikolova, N., E. Nikolov. ML-Structures in the Repetitive Robust Control Systems. - Cybernetics and Information Technologies Journal, 8, 2008, No. 1, BAS, 44-64.

18. Nikolova, N., E. Nikolov. Methods for Analysis of Robust and Filtration Features of Repetitive Control Systems - Part I, II. Journal Proceedings of the Technical University of Sofia, Publishing House of Technical University of Sofia, 65, 2015, Book 1, 2015, ISSN 1311-0829, 71-90.

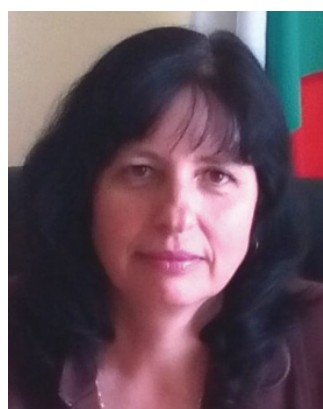

Associated Professor Nina Nikolova, Ph. D. (born 1970). Education: M.Sc (1994) Technical University of Sofia Faculty of Automation (TUS - FA). Scientific degrees: Ph. D. (2004) "Research Robust Control Systems", TU - FA; Employment: Assistant Professor, TUS - FA (2002-2003), Senior Assistant Professor, TUS - FA (2003), Associated Professor, TUS - FA (2007 - present). Career related activities: Dean of Faculty of Automation, Technical University of Sofia (2015 - present); Publications in international and Bulgarian journals -85 ; books -4 . University teaching experience: Technical University of Sofia - Faculty of Automation and Filière Francophone de Génie Electrique. Training Courses and consultation - 16. Defended students: scientific supervisor for the degree-projects of the 30 students. Scientific interests: Robust Control and Industrial Applications; Fractional Control Algorithms and Systems; Disturbances Absorbing Control; Repetitive Control Systems.

Contacts:

Technical University of Sofia Faculty of Automation Department "Industrial Automation" e-mail:ninan@tu-sofia.bg

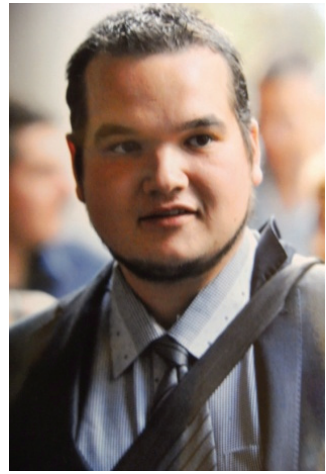

Boris Grasiani, PhD student, (born 1989). Education: finished in 2008 High School of Transport and Energy , Henry Ford "; M.Sc. (2013) Technical University of French Faculty (Faculté Francophone de Génie Electrique). Scientific interests: Robust Control Systems, Fractional Control Algorithms, Repetitive Control Systems, Automatic Control and Industrial Applications.

Contacts:

Technical University of Sofia Department "Industrial Automation" e-mail:bgrasiani@tu-sofia.bg 